\title{
Nonlinear Analytical Model of Localized Sub-THz and THz Rectifications in FET Power Detectors
}

This paper was downloaded from TechRxiv (https://www.techrxiv.org).

LICENSE

CC BY-NC-SA 4.0

SUBMISSION DATE / POSTED DATE

03-11-2021 / 06-11-2021

\section{CITATION}

elkhatib, tamer (2021): Nonlinear Analytical Model of Localized Sub-THz and THz Rectifications in FET Power Detectors. TechRxiv. Preprint. https://doi.org/10.36227/techrxiv.16923928.v1

$\mathrm{DOI}$

10.36227/techrxiv.16923928.v1 


\title{
Nonlinear Analytical Model of Localized Sub-THz and $\mathrm{THz}$ Rectifications in FET Power Detectors
}

\author{
Tamer A. Elkhatib, Member, IEEE,
}

\begin{abstract}
A nonlinear analytical model for THz FET power detectors based on their distributed RC network is presented. This empirical model works well for both drain-unbiased and drainbiased THz FET responses. The physics-based analysis reveals that localized $\mathbf{T H z}$ rectifications in long channel transistors may be mathematically expressed in the same way as regular RF frequency rectifications of a single lumped device. However, the one lumped FET model can't work properly at THz frequencies without correct definitions of $\mathrm{THz}$ signals on its terminals and independently considers localized rectifications on the source and drain sides. An improved compact one lumped THz FET power detector model with additional Schottky diodes at the source and drain terminals is presented. THz FET detector can also perform a simultaneous self-amplification (active rectification) of the localized THz rectified dc signal when operates in the saturation regime beyond its unity gain frequency. A novel analytical expression for the localized THz dc rectified response is developed for FETs operating in the saturation regime. The presented physics-based model agrees excellently with the measured experimental results of GaAs HEMT transistors at $1.6 \mathrm{THz}$ under arbitrary biasing conditions. Many novel electronic designs can be implemented for Millimeter-wave and $\mathrm{THz}$ technologies based on the physical FET's nonlinear nature in this frequency range.
\end{abstract}

Index Terms-Nonlinear analytical model, Field Effect Transistors (FETs), Plasma wave electronics, Distributed resistive FET mixer, Self-Mixing $\mathrm{THz}$ detector, Millimeter-wave mixers, Terahertz (THz) Detectors, Terahertz Rectifications, FET Power detectors, Active rectifications, TeraFETs.

\section{INTRODUCTION}

$\mathrm{T}$ ERAHERTZ and Sub-THz electronics are gaining much interest recently in many potential applications $[1,2]$. Among these applications are communication systems [3], security imaging [4], industrial quality inspection [5], biomedical research [6,7], radars or lidars [8], and material characterization [9]. A couple of decades ago, microwave nonlinear electronic sources and detectors were only used at Sub-THz and THz ranges $[10,11]$ with limited source powers or detection responsivities that degrade quickly with frequency $(f)$ at the rate of $\left(f^{-2}\right)$. Recently, the maximum unity gain

This paragraph of the first footnote will contain the date on which you submitted your paper for review. It will also contain support information, including sponsor and financial support acknowledgment. For example, "This work was supported in part by the U.S. Department of Commerce under Grant BS123456." frequency $\left(f_{t}\right)$ and the maximum oscillation frequency $\left(f_{\max }\right)$ of some semiconductor technologies are being pushed deeper into the Sub-THz and $\mathrm{THz}$ ranges. Therefore, there are many emerging transistor technologies capable of $\mathrm{THz}$ amplifications nowadays [12].

In the '90s, Dyakonov and Shur [13-15] proposed a different method to handle the nonlinearity of FET devices at higher frequencies especially near and beyond their cut-off frequencies. Dyakonov-Shur's plasma-wave concept is equivalent physically to the approach of modeling the channel of FETs as a distributed RC or RLC transmission line network [16-18]. The non-resonant $\mathrm{THz}$ response (governed by overdamped ac oscillations or by distributed resistive self-mixing) has demonstrated that FETs can achieve $\mathrm{THz}$ responsivity much higher than traditional microwave nonlinear electronic devices [19].

Although there are few analytical solutions for the diffusion equation describing the distributed RC network problem, numerical methods can handle this problem in a better way $[20,21]$. Many approximate analytical formulas [22,23] have been proposed for the zero-drain biased FETs as non-resonant $\mathrm{THz}$ detectors. At the zero-drain bias condition, the distributed $\mathrm{RC}$ network can be assumed to be uniform along the entire channel with identical distributed $\mathrm{R}$ and $\mathrm{C}$ elements. While at drain-bias condition, the transistor channel RC distributed network is not uniform anymore, hence an approximate analytical solution becomes more difficult, and only numerical simulations can model the transmission line behavior of FET's channel $[24,25]$. In most of the published research work, coldFETs (zero-biased) are used as square-law THz power detectors [26]. On the other hand, few works were reported on the drainbiased THz FET detectors with only phenomenological models that are not physics-based [27-29].

In this work, an analytical nonlinear model is developed based on the distributed FETs nature at $\mathrm{Sub}-\mathrm{THz}$ and $\mathrm{THz}$ frequencies for both drain-biased and drain-unbiased conditions. It is demonstrated here that all reported analytical models can be directly derived from this general physics-based nonlinearity analytical model. It is also demonstrated here that the one

T. A. Elkhatib is with the Department of Engineering Mathematics and Physics, Faculty of Engineering, Cairo University, Giza, 12136, Egypt. (e-mail: t.a.elkhatib@cu.edu.eg). 
lumped FET approximation at $\mathrm{THz}$ frequencies can obtain only an equivalent mathematical expression for $\mathrm{THz}$ rectifications governed by the nonlinearity of the device's static I-V characteristics as suggested by Refs. [30,31]. However, the one lumped model cannot account for the physical localization and the independent $\mathrm{THz}$ rectification at the source and drain sides of the THz FET's channel. Hence, the one lumped FET approximation results in multiple simulation errors that are explained by the developed analytical model in this work. It is justified here that the gate-source and gate-drain edges can be logically considered as ultra-fast higher-frequency Schottky diode terminals. As a result, an improved one lumped THz FET model is proposed with additional rectifying Schottky diodes at the source and drain terminals of the THz FET power detector.

For the drain-biased FET detectors and especially when FET is operating in the saturation regime, the localized $\mathrm{THz} \mathrm{dc}-$ rectifications at the gate-source and gate-drain edges can be simultaneously amplified by many orders of magnitude based on the amplification configuration of the circuit connectivity for the used THz FET detector [32]. In the case of a common source amplifier configuration, the gate-source localized $\mathrm{THz}$ dcrectification is amplified at the drain side with orders of magnitude, while the gate-drain localized $\mathrm{THz}$ dc-rectifications become completely negligible. The developed analytical model here shows much better agreement than other commonly known models when compared with room-temperature experimental results of $1.6 \mathrm{THz}$ detection using $(80 \mu \mathrm{m} \times 500 \mathrm{~nm}) \mathrm{GaAs}$ HEMT transistors [33].

This paper is organized as follows: Section II presents a nonlinear analytical model for relatively long channel zerobiased distributed THz FET detectors. Section III extends the developed analytical model for arbitrary drain-biasing conditions. The nonlinearity model is then validated in Section IV by excellent agreement with experimental results. Section IV also discusses a comparison between the analytical model of this work and the other simplified models commonly reported in the THz community. Finally, section VI draws the main conclusion.

\section{ZERO-DRAIN BIASED THZ FET DETECTORS}

Near and beyond cutoff frequency, the distributed nature of FET transistors needs to be considered [24,25]. Some numerical $\mathrm{THz}$ Spice models have been proposed based on the distributed FET network [20]. On the other hand, few analytical models have been commonly used based on the non-resonant detection model of overdamped plasma-wave oscillation [22,23], or based on distributed resistive FET self-mixing [26], or based on localized self-mixing technique [34,35]. All the available analytical models can behaviorally describe the FETs THz dc rectification, but they are not precise models yet. They either neglect the complete nonlinearity effects in the THz FET detectors, or they are using one lumped FET model that is not physics-based [30,31]. Here, a physics-based analytical model starting from the distributed FET model will be developed that is more general and precise.
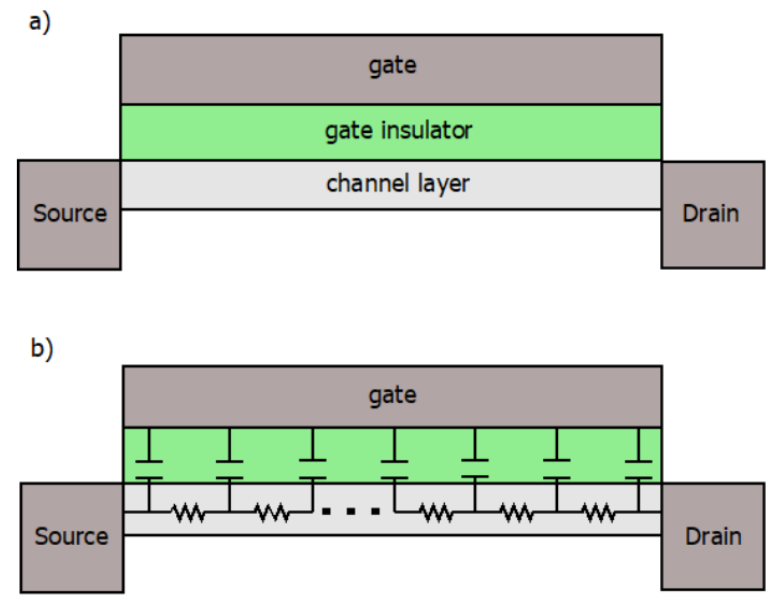

c)

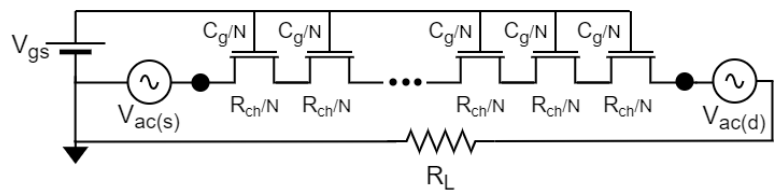

Fig. 1. The structure of a FET (a) and its equivalent distributed RC transmission line model (b) for $\mathrm{THz}$ detection. An additional distributed $\mathrm{THz}$ FET model (c) for numerical circuit simulations.

Fig. 1 presents the distributed network models for THz FET detectors. Since this paper focuses only on the non-resonant THz FET's responses, the Drude inductance [21] is neglected and only the RC model is considered. The diffusion equation (1) describes analytically the voltage $V(x, t)$ across the distributed RC channel in Fig. 1(b). Equation (1) is equivalent to solving the hydrodynamic equations from device physics (Euler and Continuity) that is used in the non-resonant plasmawave model [23].

$$
\left(\frac{R_{c h}}{N} \frac{C_{g}}{N}\right) \frac{\partial V(x, t)}{\partial t}=\frac{\partial^{2} V(x, t)}{\partial x^{2}}
$$

Where $R_{c h}$ is the total transistor channel resistance, $C_{g}$ is the total gate-to-channel capacitance, and both $R_{c h}\left(V_{g s}\right)$ and $C_{g}\left(V_{g s}\right)$ are a function of the applied gate-source voltage and $N$ is the number of segmentations used in the numerical solver. In this work, the distributed FET network in Fig.1 (c) will be adapted for simplicity to derive a general analytical model for the case of high-frequency operation and long-channel FETs. It is assumed here that $N$ is large enough such that the quantization of $V(x, t)$ is considered as a continuous function along the transistor channel $[20,24]$.

Assuming that two $\mathrm{THz}$ ac signals are applied on both source $V_{a c(s)}$ and drain $V_{a c(d)}$ terminals. At frequencies much below the cutoff frequency, the ac signal will be uniform across the transistor channel with no spatial dependence. At higher frequencies, one approximate solution of (1) can be found as $V_{s}(x) V_{a c(s)} \sin \left(\omega t-x / \sqrt{2} L_{o}\right)$, where $V_{s}(x)=\exp \left(-x / \sqrt{2} L_{o}\right)$ 
is an exponentially decaying expression across the transistor channel starting from the source edge [23]. Similarly, $V_{d}(x) V_{a c(d)} \sin \left(\omega t+\left(x-L_{c h}\right) / \sqrt{2} L_{o}\right)$ exists near the drain edge, where $V_{d}(x)=\exp \left(\left(x-L_{c h}\right) / \sqrt{2} L_{o}\right) \quad$ is another exponentially decaying expression from the drain edge, while $L_{c h}$ is the transistor's total channel length, and $L_{o}$ is defined as an ac characteristic propagation length inside the distributed FET network. The definition of this characteristic propagation length may vary from one model to another $[20,23]$, but the final rectification solution is independent of it. An approximate expression for $L_{o}$ is given here by: $L_{o}=\sqrt{L_{c h}^{2} / \omega R_{c h} C_{g}}$.

On the other hand, a numerical circuit solver has been used with many segments as in Fig. 1(c) and it was found numerically that the ac voltage degrades linearly within the elements inside the distributed FET network as shown in Fig. 2. To match between the numerical solver and the analytical approximation, the exponential decaying expression can be approximated as $\exp (x)=1+x$.

For a high-frequency and long channel device $\left(L_{o}<<L_{c h}\right)$, the ac signal is localized only near the transistor channel edges from the source and drain sides. As a result of these localized ac signals, a total induced current (both $\mathrm{AC}$ and DC) is generated in the distributed device and it can be expressed by Taylor series expansion as:

$$
\begin{aligned}
I(x, t)= & N\left[\frac{\partial I_{d s}}{\partial V_{g s}} \partial V_{g s}(x, t)+\frac{\partial I_{d s}}{\partial V_{d s}} \partial V_{d s}(x, t)\right. \\
& +\frac{1}{2} \frac{\partial^{2} I_{d s}}{\partial V_{g s}{ }^{2}} \partial V_{g s}{ }^{2}(x, t)+\frac{1}{2} \frac{\partial^{2} I_{d s}}{\partial V_{d s}{ }^{2}} \partial V_{d s}{ }^{2}(x, t) \\
& \left.+\frac{\partial^{2} I_{d s}}{\partial V_{g s} \partial V_{d s}} \partial V_{g s}(x, t) \partial V_{d s}(x, t)+\ldots\right]
\end{aligned}
$$

Where $I_{d s}$ is the total static drain-source current within the entire distributed device, $V_{g s}$ is the static gate-to-source voltage applied on the entire device, $V_{d s}$ is the very small static drainto-source voltage since there is zero-drain biasing on the device. While $\partial V_{g s}(x, t)=\partial V_{d s}(x, t)=-V(x, t)$ are the identical applied $\mathrm{THz}$ ac signals on the distributed elements of the FET network near the source and drain sides as described in Fig. 1(c). To find the localized dc-voltage rectified at each edge of the transistor's channel independently, one can take the time average for the current expression at (2) multiplied by the local channel resistance such that:

$$
V_{d s(i n d)}(x)=\frac{1}{2 \pi} \int_{\omega t=0}^{\omega t=2 \pi} I(x, t) \times\left(\frac{R_{c h}}{N}\right) \quad 0<x<L_{c h}
$$

It is important to notice that the locally induced dc-current at the source side will flow in the positive direction from drain to source leading to a source potential lower than that of the drain

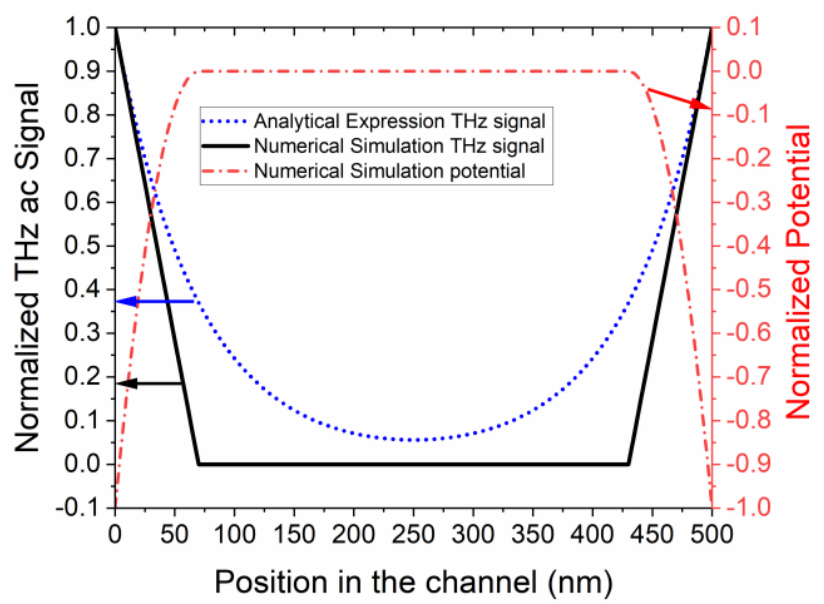

Fig. 2. The analytical and numerical normalized $1.6 \mathrm{THz}$ ac oscillations across the distributed FET's channel (Left) and its corresponding normalized induced $\mathrm{THz}$ dc potential (Right). The FET's room-temperature parameters at zerobiasing condition are $L_{c h}=500 \mathrm{~nm}, \quad R_{c h}=10 \mathrm{KOhms}$ at $V_{g s}=0.37 \mathrm{~V}$, $C_{g}=1$ Femtofarads, $L_{o}=50 \mathrm{~nm}$.

side [35]. In contrast to this, the locally induced dc-current at the drain side will flow in the negative direction from source to drain leading to a drain potential lower than that of the source side as shown in Fig. 2.

Only the quadratic terms will contribute to the rectified dc response from (2) after substituting in (3). The total maximum induced drain-source de voltage $\Delta V_{d s}^{\text {unbiased }}$ can be found as:

$$
\begin{aligned}
& \Delta V_{d s}^{\text {unbiased }}=V_{d s(\text { ind })}\left(x=L_{c h}\right)-V_{d s(i n d)}(x=0) \\
& =\left(\frac{V_{s(a c)}^{2}-V_{d(a c)}^{2}}{2}\right) \times\left(\frac{1}{2} \frac{\partial^{2} I_{d s}}{\partial V_{g s}{ }^{2}}+\frac{1}{2} \frac{\partial^{2} I_{d s}}{\partial V_{d s}{ }^{2}}+\frac{\partial^{2} I_{d s}}{\partial V_{g s} \partial V_{d s}}\right) R_{c h}
\end{aligned}
$$

Where all the terms in (4) are calculated from the entire FET static I-V characteristics as one lumped element with no dependence on the number of segments $N$ used in the model or the ac characteristics propagation length $L_{o}$. Hence, one can surmise that the localized Sub-THz or $\mathrm{THz}$ rectifications inside the distributed FET channel can be calculated mathematically in a similar way to the rectifications of the much lower ac frequency applied on the FET terminals in the RF range [31]. Moreover, using a transistor distributed RC network model is redundant as suggested in Refs. [30].

However, there are few important differences between lowfrequency and high-frequency rectification that worth to be mentioned here. The first difference is that the dc rectifications are localized at channel edges simultaneously and they are independently compensating each other reducing the total induced THz FET response. For such compensation effect, many designs have been reported [36,37] developing an unsymmetric condition between both drain and source edges to maximize the overall $\mathrm{THz}$ response. If once confusedly consider the THz FET detector as one lumped device [31], then $\partial V_{g s}=-V_{a c(s)} \sin \omega t$ and $\partial V_{d s}=\left(V_{a c(d)}-V_{a c(s)}\right) \sin \omega t$, and the one lumped THz FET detector produces a completely different rectified signal from the real response described by (4) such as: 


$$
\begin{aligned}
& \Delta V_{d s(\text { lumped })}^{\text {unbiased }}=\left(\frac{V_{a c(s)}^{2}}{2}\right) \times\left(\frac{1}{2} \frac{\partial^{2} I_{d s}}{\partial V_{g s}^{2}}\right) R_{c h} \\
& +\left(\frac{\left(V_{a c(d)}-V_{a c(s)}\right)^{2}}{2}\right) \times\left(\frac{1}{2} \frac{\partial^{2} I_{d s}}{\partial V_{d s}{ }^{2}}\right) R_{c h} \\
& +\left(\frac{\left(V_{a c(d)}-V_{a c(s)}\right) \times\left(-V_{a c(s)}\right)}{2}\right)\left(\frac{\partial^{2} I_{d s}}{\partial V_{g s} \partial V_{d s}}\right) R_{c h}
\end{aligned}
$$

Coincidentally, the one lumped FET model (5) and the distributed FET model (4) do converge mathematically into the same solution if only the localized THz signal is independently considered at the source side. Physically, the one lumped model still can't account for the localization of $\mathrm{THz}$ rectifications near the edges of the FET channel.

Another significant difference is the frequency response of the one lumped model versus the distributed RC model. The maximum $\mathrm{THz}$ response of the one lumped FET is expected to degrade as the applied frequency increases towards higher $\mathrm{THz}$ frequencies. It is expected that the maximum detected response in (5) will have a roll-off of $20 \mathrm{~dB} /$ decade as described in Ref. [30]. This frequency roll-off is of a typical RC time constant nature where $\mathrm{R}$ is approximately the transistor's total channel resistance $R_{c h}$ and $\mathrm{C}$ is the total gate capacitance $C_{g}$. On the contrary, the ideal frequency response of the distributed RC FET model degrades less with frequency when compared with that of the one lumped FET model as proved experimentally in Refs. [22] and analytically in Ref. [38]. The localized THz rectifications occupy only a fraction of the distributed FET total channel. Hence, the effective resistance is approximately given by $R_{c h} \times \sqrt{2} L_{o} / L_{c h}$ and the effective capacitance is approximately given by $C_{g} \times \sqrt{2} L_{o} / L_{c h}$, and both decrease as frequency increases. Therefore, the distributed FET model is much faster and does operate much better at higher frequencies than the one lumped FET model.

In practical experiments, the $\mathrm{THz}$ signal is typically not wired directly to transistors terminals [39], instead $\mathrm{THz}$ radiation is falling on the transistor surface. $\mathrm{THz}$ radiation is coupled either through the chip bonding wires, chip metallization layers or in much better situations through an integrated chip antenna [40-43]. In general, the frequency dependence of the non-resonant THz FET detectors is depending on the frequency coupling characteristics of the connecting wires and chip metallization or the integrated antenna. Hence, one can observe experimentally different frequency dependencies based on each design configuration.

It is important to notice that distributed $\mathrm{THz}$ FET detectors don't only rectify the applied ac signals between the drain-tosource terminals, it simultaneously rectifies across the gate-tosource and gate-to-drain terminals as follows:

$$
\begin{aligned}
& \Delta V_{g s}^{\text {unbiased }}=V_{d s(i n d)}(x=0) \\
& \quad=\left(\frac{V_{s(a c)}^{2}}{2}\right) \times\left(\frac{1}{2} \frac{\partial^{2} I_{d s}}{\partial V_{g s}{ }^{2}}+\frac{1}{2} \frac{\partial^{2} I_{d s}}{\partial V_{d s}{ }^{2}}+\frac{\partial^{2} I_{d s}}{\partial V_{g s} \partial V_{d s}}\right) R_{c h}
\end{aligned}
$$

$$
\begin{aligned}
& \Delta V_{g d}^{\text {unbiased }}=V_{d s(\text { ind })}\left(x=L_{c h}\right) \\
& \quad=\left(\frac{V_{d(a c)}^{2}}{2}\right) \times\left(\frac{1}{2} \frac{\partial^{2} I_{d s}}{\partial V_{g d}{ }^{2}}+\frac{1}{2} \frac{\partial^{2} I_{d s}}{\partial V_{d s}{ }^{2}}+\frac{\partial^{2} I_{d s}}{\partial V_{g d} \partial V_{d s}}\right) R_{c h}
\end{aligned}
$$

Equations (6) and (7) can be correlated to the total dc rectified signal across the transistor's channel in (4) as follows: $\Delta V_{d s}^{\text {unbiased }}=\Delta V_{g s}^{\text {unbiased }}-\Delta V_{g d}^{\text {unbiased }}$. However, one must consider that the applied gate-to-source and gate-to-drain voltages are almost identical $\left(V_{g s}=V_{g d}\right)$ in the zero-drain biasing condition, therefore the terms like $\partial^{2} I_{d s} / \partial V_{g s}^{2}, \partial^{2} I_{d s} / \partial V_{g d}^{2}$ are also identical in this configuration.

In the case of using a loading resistor $R_{L}$ connected between the transistor drain and source terminals [44], the simple voltage divider equation can be used and the maximum induced $\mathrm{THz}$ dc response with load $\Delta V_{d s(l o a d)}^{\text {unbiased }}$ can be expressed as:

$$
\Delta V_{d s(\text { load })}^{\text {unbiased }}=\Delta V_{d s}^{\text {unbiased }} \times \frac{R_{L}}{R_{L}+R_{c h}}
$$

One possible approximation is to takes the spatial average of the induced dc rectifications $V_{d s(\text { ind })}(x)$ in (3) near the drain and source edges independently as follows:

$$
\begin{aligned}
& V_{d s \text { (ind-source })}=\frac{1}{\sqrt{2} L_{o}} \int_{0}^{\sqrt{2} L_{o}} V_{d s(\text { ind })}(x) \\
& V_{d s \text { (ind-drain) }}=\frac{1}{\sqrt{2} L_{o}} \int_{L_{c h}-\sqrt{2} L_{o}}^{L_{c h}} V_{d s(\text { ind })}(x)
\end{aligned}
$$

By using (9) and (10), the analytical expressions for the induced THz FET's dc responses given by (4), (6), and (7) need to be scaled by a minor factor of approximately a half.

By deeply investigating the three second-order terms in the derived dc induced THz responses of the distributed FETs; one can find that the two second-order terms $\partial^{2} I_{d s} / \partial V_{d s}{ }^{2}$ and $\partial^{2} I_{d s} / \partial V_{g s}{ }^{2}$ are generally much smaller than the other term $\partial^{2} I_{d s} / \partial V_{g s} \partial V_{d s}$ for a zero-drain biasing condition. However, for an optimized THz FET detector with high responsivity, all the three $2^{\text {nd }}$ order terms should be considered as shown later in the validation of the model results in Section IV. All three terms are comparable for some applied dc gate-to-source values (especially near the peak values of THz FET response). On the contrary, most of the reported analytical models by the $\mathrm{THz}$ community only consider the term $\partial G_{c h} / \partial V_{g s}$ and neglects the other $2^{\text {nd }}$ order terms [22,23], where the channel conductance is defined as $G_{c h}=\partial I_{d s} / \partial V_{d s}$. Hence, a simplified behavioral model for THz FET detectors can be given as:

$$
\Delta V_{d s(l o a d)}^{\text {unbiased }}=\left(\frac{V_{s(a c)}^{2}-V_{d(a c)}^{2}}{4}\right) \times\left(\frac{\partial G_{c h}}{\partial V_{g s}} \frac{1}{G_{c h}}\right)\left(\frac{R_{L}}{R_{c h}+R_{L}}\right)
$$


The latest equation introduces a significant technical meaning of the induced $\mathrm{THz}$ rectifications at both source and drain edges of the used THz FET detectors. Equation (11) is showing a response dependence that is like the response of the Schottky diode detectors given by: $\left(\partial^{2} I / \partial^{2} V\right) \times(\partial V / \partial I)$. Such similarity makes one considers the FET's channel edges as logically induced Schottky diodes that operate as ultra-fast higher-frequency rectifiers and mixers in the Sub- $\mathrm{THz}$ and $\mathrm{THz}$ ranges. However, THz FETs have the main advantage over the regular Schottky diodes which is the capability of tuning the channel resistance with the applied gate-source voltage.

To end the confusion between the (quasi-static) one lumped FET model versus the (non-quasi-static) distributed FET model, an improved compact one lumped FET model for THz power detectors is proposed based on the nonlinearity analysis done before. Fig. 3 presents the basic one lumped FET device model with only an additional two Schottky diodes [45] at both source and drain terminals. It is assumed that the localized $\mathrm{THz}$ ac signals are only applied on the additional Schottky diodes which are completely transparent for any applied dc biasing.
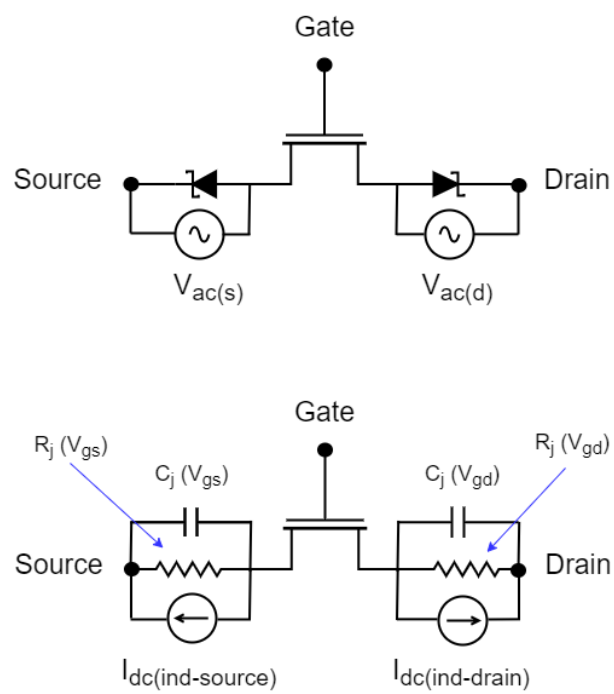

Fig. 3. An improved one lumped model for THz FET power detectors with additional Schottky diodes at source and drain sides (Top), and with the diodes' small-signal equivalent circuit as well as the localized induced $\mathrm{THz}$ rectified dc-current (Bottom).

The two independent Schottky diodes have junction resistance and capacitance that are defined only in the localized $\mathrm{THz}$ ac rectification distance as: $R_{j}\left(V_{g s}\right)=R_{c h} \times \sqrt{2} L_{o} / L_{c h}$, $C_{j}\left(V_{g s}\right)=C_{g} \times \sqrt{2} L_{o} / L_{c h}$ near the source side and similarly $R_{j}\left(V_{g d}\right)$ and $C_{j}\left(V_{g d}\right)$ near the drain side. The location of the additional Schottky diodes within the improved lumped FET model reflects the induced de signals across both drain-tosource and gate-to-source responses simultaneously on the source side and similarly across drain-to-source and gate-todrain responses at the drain side. This improved one lumped THz FET model perfectly matches the nonlinear analytical model of the distributed THz FET power detector and it works for both zero-drain biased FET detectors and under any arbitrary drain biasing as will be shown in the following section.

\section{DRAIN-BIASED THZ FET DETECTORS}

By applying drain bias, the THz FET detector is derived to operate in the triode region and later into the saturation regime. The distributed $R_{n}(x)$ and $C_{n}(x)$ elements in the FET transmission line model are not uniform anymore as shown in Fig. 4. On the drain side, the channel local resistance starts to increase as $V_{d s}$ increases and simultaneously $V_{g d}$ decreases.

The ac propagation length at the drain side starts to reduce and especially when the transistor starts operating in the saturation regime when $V_{d s}>\left(V_{g s}-V_{t h}\right)$. On the other hand, the rectifications on the source side are minimally affected by the drain bias and it can approximately be treated analytically the same way as the rectifications of zero-drain biased condition at the same applied gate-to-source voltage.

a)

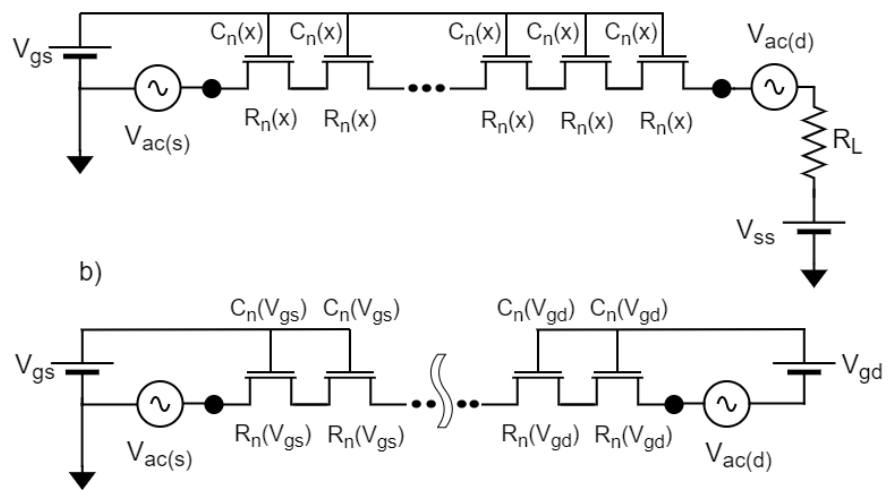

Fig. 4. A distributed THz FET circuit model (a) under arbitrary biasing condition with non-uniform distributed elements. An equivalent two quasiuniform distributed THz FET circuit models (b) that are independent at the source and drain sides

Generally, it is more difficult to find a closed-form analytical solution for the diffusion equation with non-uniform $\mathrm{RC}$ elements. However, the problem can be divided into two simplified circuit models: (one model at the source edge and another independent model at the drain edge) and a superposition concept can be used to add the two solutions.

Firstly, one can consider that a distributed FET network near the source side with quasi-uniform RC elements that depend only on the applied gate-to-source voltage as in Fig. 4(b). The applied $V_{d s}$ voltage to these distributed elements is negligible as in the zero-drain biased case. Hence the first rectified solution $\Delta V_{d s(1)}^{\text {biased }}$ near the source side can be expressed the same as the drain-unbiased case such that:

$$
\begin{aligned}
& \Delta V_{d s(1)}^{\text {biased }}=\Delta V_{g s}^{\text {biased }}=\Delta V_{g s}^{\text {unbiased }} \\
& =\left(\frac{V_{s(a c)}^{2}}{2}\right) \times\left.\left(\frac{1}{2} \frac{\partial g_{m}}{\partial V_{g s}}+\frac{1}{2} \frac{\partial G_{c h}}{\partial V_{d s}}+\frac{\partial G_{c h}}{\partial V_{g s}}\right) R_{c h}\right|_{V_{d s} \approx 0}
\end{aligned}
$$

Secondly, one can consider another independent distributed FET network near the drain side with quasi-uniform RC elements that depend only on the applied gate-to-drain voltage 
as in Fig. 4(b). Also, the applied $V_{d s}$ voltage to these distributed elements is still negligible as in the zero-drain-biased case. Hence, the second rectified solution $\Delta V_{d s(2)}^{\text {biased }}$ can be expressed as:

$$
\begin{aligned}
& \Delta V_{d s(2)}^{\text {biased }}=-\Delta V_{g d}^{\text {biased }}=-\Delta V_{g d}^{\text {unbiased }} \\
& =\left(\frac{-V_{d(a c)}^{2}}{2}\right) \times\left.\left(\frac{1}{2} \frac{\partial g_{m}}{\partial V_{g d}}+\frac{1}{2} \frac{\partial G_{c h}}{\partial V_{d s}}+\frac{\partial G_{c h}}{\partial V_{g d}}\right) R_{c h}\right|_{V_{d s} \approx 0}
\end{aligned}
$$

So far, the induced THz FET responses on the gate-to-source and the gate-to-drain terminals look very similar for both drainbiased and drain-unbiased conditions. The main difference is that $V_{g s}$ and $V_{g d}$ are equals in the zero-drain biasing case, however, they are different (and treated independently) in the drain-biased case. Therefore, the improved one lumped $\mathrm{THz}$ FET model with additional two Schottky diodes at the source and drain terminals is still working well for arbitrary $V_{g s}$ and $V_{d s}$ applied voltages.

Unexpectedly, the total induced drain-to-source voltage is not just the superposition of the induced two expressions (12) and (13). There is an additional generated THz response that is induced as a secondary effect from the complementary transistor's amplifier structure. When a transistor is drainbiased, any induced de variation on its gate-to-source terminals will be simultaneously amplified at the drain side such that: $\Delta V_{d s(3)}^{\text {biased }}=\Delta V_{g s}^{\text {biased }}\left(\partial V_{d s} / \partial V_{g s}\right)$. At very small biased $V_{d s}$ voltages and in a triode operation mode, this amplification gain $\partial V_{d s} / \partial V_{g s}$ is almost negligible. However, this amplification gain starts to increase by orders of magnitude when the transistor operates in the saturation regime. On the other hand, the induced $\mathrm{dc}$ variations on the gate-to-drain terminals are reflected as it is on the drain side since $\partial V_{d s} / \partial V_{g d}=-1$ for a fixed $V_{g s}$ voltage. Therefore, the total induced drain-to-source THz FET response including the loading effect consists of three complementary components and can be expressed as follows:

$$
\begin{aligned}
& \Delta V_{d s(\text { total })}^{\text {biased }}=\left(\Delta V_{d s(1)}^{\text {biased }}+\Delta V_{d s(2)}^{\text {biased }}+\Delta V_{d s(3)}^{\text {biased }}\right) \frac{R_{L}}{R_{L}+R_{c h}^{*}} \\
& =\left[\Delta V_{g s}^{\text {biased }}\left(1+\frac{\partial V_{d s}}{\partial V_{g s}}\right)-\Delta V_{g d}^{\text {biased }}\right] \frac{R_{L}}{R_{L}+R_{c h}^{*}} \\
& =\left[\Delta V_{g s}^{\text {biased }}\left(1-g_{m}^{*} \times R_{c h}^{*}\right)-\Delta V_{g d}^{\text {biased }}\right] \frac{R_{L}}{R_{L}+R_{c h}^{*}}
\end{aligned}
$$

Where $\Delta V_{g s}^{\text {biased }}$ and $\Delta V_{g d}^{\text {biased }}$ are defined by (12) and (13) respectively. While $R_{c h}^{*}$ and $g_{m}^{*}$ in (14) are calculated from the lumped FET static I-V characteristics under arbitrary applied $V_{g s}$ and $V_{d s}$ voltages. When the THz FET detector is biased in the saturation regime only, the analytical expression given by (16) can be approximated as:

$$
\Delta V_{d s \text { (total })}^{\text {biased }}=-\Delta V_{g s}^{\text {biased }} g_{m}^{*} \times \frac{R_{c h}^{*} \times R_{L}}{R_{L}+R_{c h}^{*}}
$$

This amplification scheme is also covered with the improved one lumped THz FET power detector model with the additional Schottky diodes presented in Fig. 3. The improved lumped model also supports all other amplification schemes rather than the common source amplifier used in this analysis [32].

The THz FET response given by (15) has been compared with the quasi-static $2^{\text {nd }}$ order rectifications of the one lumped FET model under arbitrary biasing conditions. Since the localized rectifications at the source side are only significant at the saturation regime biasing condition. Also, only the term $\partial g_{m}^{*} / \partial V_{g s}$ has a significant contribution to the rectified signal neglecting the other $2^{\text {nd }}$ order terms. Hence, the one lumped FET model does converge again mathematically with the distributed FET model, and (15) can be rewritten as:

$$
\Delta V_{d s(\text { total })}^{\text {biased }}=-\left(\frac{V_{s(a c)}^{2}}{4}\right) \frac{\partial g_{m}^{*}}{\partial V_{g s}} \times \frac{R_{c h}^{*} \times R_{L}}{R_{L}+R_{c h}^{*}}
$$

However, the quasi-static nonlinearity analysis of the one lumped FET model can't physically explain the reported positive and negative responses that appear at small drain-bias values [46]. On the contrary, equation (14) based on the distributed FET model can account for such real THz FET responses. As a result of all the above, one can deduce a novel analytical expression of the localized $\mathrm{THz}$ rectifications at the source side for THz FET detectors operating in the saturation regime by comparing (17) and (18) as follows:

$$
\begin{aligned}
\Delta V_{g s}^{\text {biased }} & =\left(\frac{V_{s(a c)}^{2}}{4}\right) \frac{\partial^{2} I_{d s}}{\partial V_{g s}^{2}} \times \frac{\partial V_{g s}}{\partial I_{d s}}=\left(\frac{V_{s(a c)}^{2}}{4}\right) \frac{\partial g_{m}^{*}}{\partial V_{g s}} \times \frac{1}{g_{m}^{*}} \\
& =\left(\frac{V_{s(a c)}^{2}}{4}\right) \frac{\partial \ln \left(g_{m}^{*}\right)}{\partial V_{g s}}
\end{aligned}
$$

This latest analytical expression verifies the earlier assumption that the localized $\mathrm{THz}$ rectifications at the transistor's source side are completely independent of the application of drain-to-source biasing voltages as the expression $\partial \ln \left(g_{m}^{*}\right) / \partial V_{g s}$ is just a constant term for a fixed applied gate-to-source voltage in the saturation regime.

\section{Model VAlidation}

In general, all the information needed to calculate the induced THz FETs response can be obtained from the FET's static I-V characteristics (opposite to the belief in [38]). The validity of the developed analytical model here is established through comparison with $1.6 \mathrm{THz}$ detection results presented in Ref. [33]. The transistor used experimentally is a relatively long channel InGaAs/GaAs HEMT transistor with a $500 \mathrm{~nm}$ channel length. Fig. 5 shows a comparison between the measured DC characteristics and its equivalent spice fitting model. 

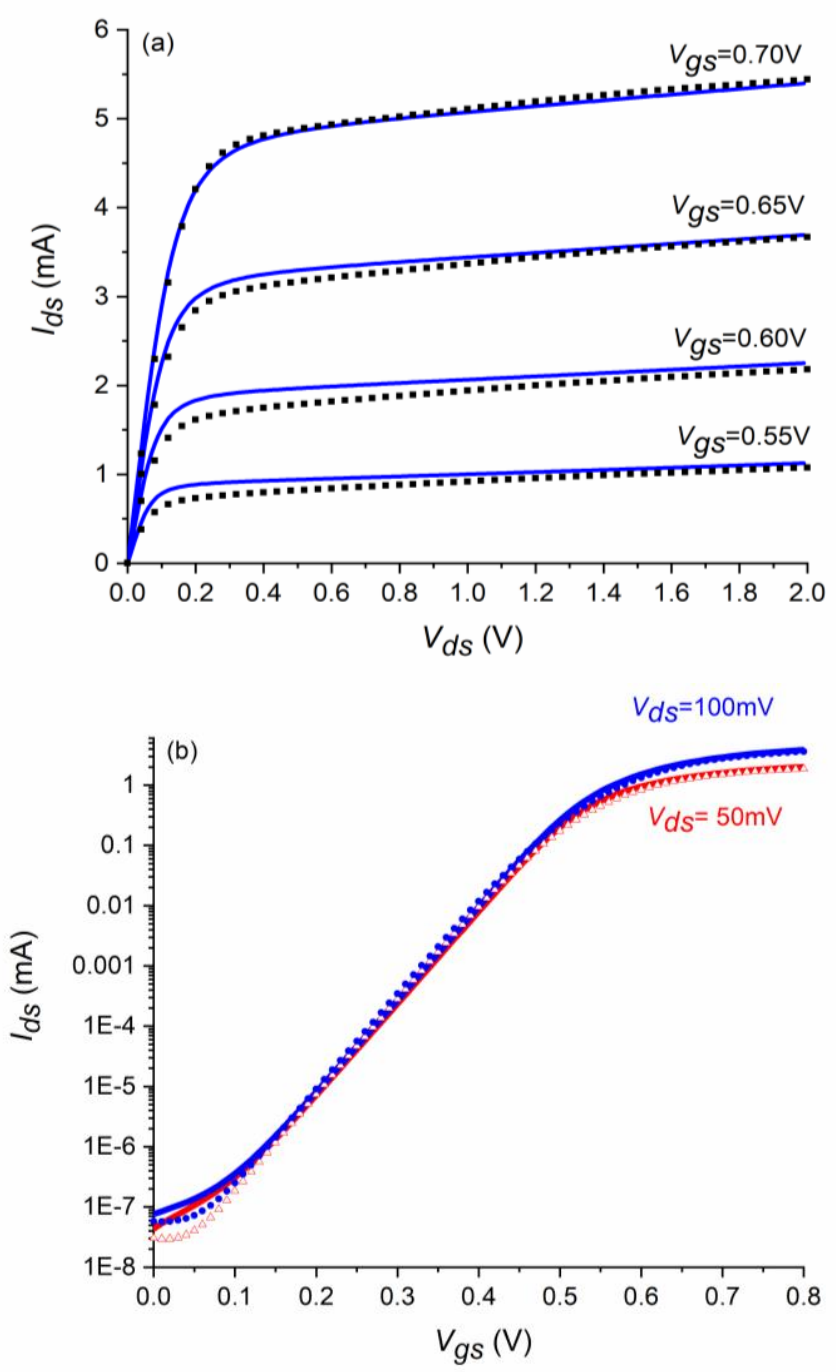

Fig. 5. Measured (dotted lines) and calculated (solid lines) DC I-V characteristics of the used GaAs HEMTs.

Transistor's channel resistance $R_{c h}$, as well as all $2^{\text {nd }}$ order nonlinearity terms $\partial G_{c h} / \partial V_{g s}, \partial G_{c h} / \partial V_{d s}$ and $\partial g_{m} / \partial V_{g s}$ are presented in Fig.6 as a function of the applied gate bias voltages (while the drain bias voltage is fixed at $10 \mathrm{mV}$ resembling the zero-drain applied case). Near and below the threshold voltage, all the $2^{\text {nd }}$ order terms are comparable and must be considered to calculate the total induced $\mathrm{THz}$ response. The normalized $1.6 \mathrm{THz}$ experimental HEMT response compared with the developed nonlinear analytical model that was given by (4) and (8) are shown in Fig. 7 revealing excellent agreement between theory and experiment. The simplified behavioral model described by (11) (which only considers the $\partial G_{c h} / \partial V_{g s}$ secondorder term and neglects the other terms) is also shown in Fig.7. This simplified behavioral model has less matching curve with experimental results showing a shifted peak position for $\mathrm{THz}$ response and a wider curve than experimental data.

The simplified behavioral model given by (11) has many different analytical forms that are commonly reported in the $\mathrm{THz}$ community $[23,32]$ and they are all physically equivalents to each other such that:

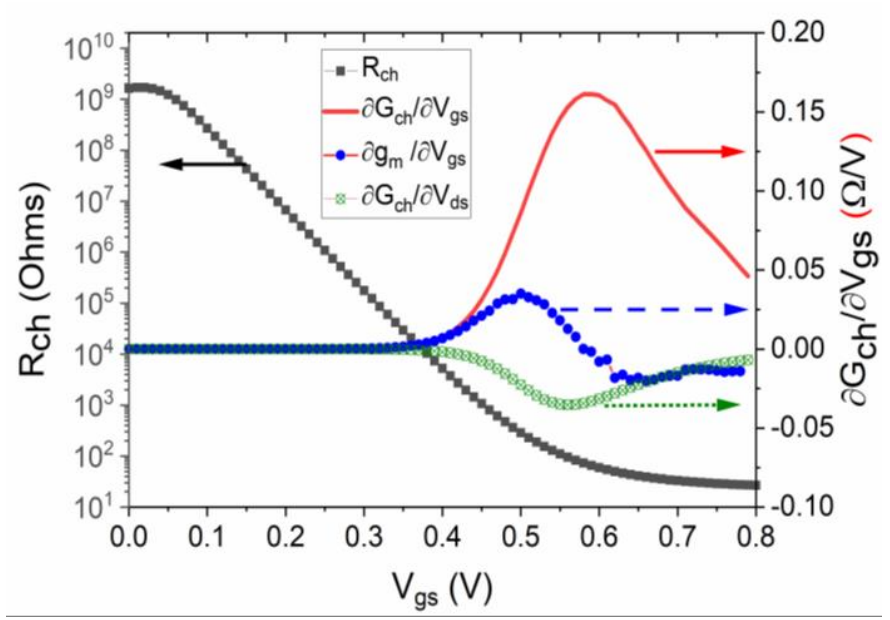

Fig. 6. The calculated HEMT channel resistance $R_{c h}$ (Left) and all $2^{\text {nd }}$ order nonlinearity terms (Right) were obtained from device DC characteristics with $10 \mathrm{mV}$ applied drain-to-source voltage.

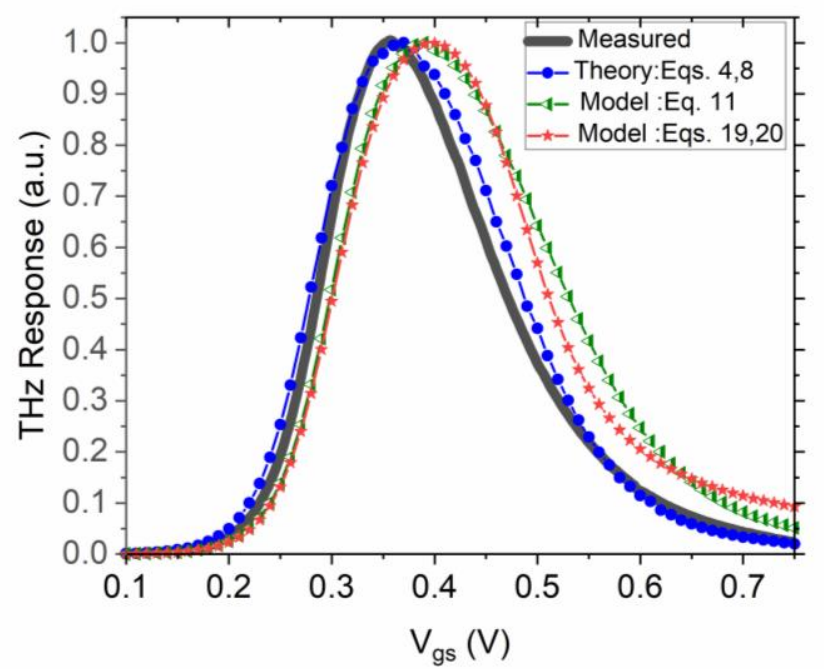

Fig. 7. The normalized non-resonant measured $1.6 \mathrm{THz}$ response of GaAs HEMT with $100 \mathrm{KOhms}$ load resistance as a function of the applied gate bias, as well as the developed nonlinear analytical model and other commonly used simplified models.

$$
\begin{aligned}
\Delta V_{d s(l o a d)}^{\text {unbiased }} & =\left(\frac{V_{s(a c)}^{2}-V_{d(a c)}^{2}}{4}\right) \times\left(\frac{\partial\left(\ln G_{c h}\right)}{\partial V_{g s}}\right)\left(\frac{R_{L}}{R_{c h}+R_{L}}\right) \\
& =\left(\frac{V_{s(a c)}^{2}-V_{d(a c)}^{2}}{4}\right) \times\left(\frac{\partial\left(\ln I_{d s}\right)}{\partial V_{g s}}\right)\left(\frac{R_{L}}{R_{c h}+R_{L}}\right) \\
& =\left(\frac{V_{s(a c)}^{2}-V_{d(a c)}^{2}}{4}\right) \times\left(\frac{g_{m}}{I_{d s}}\right)\left(\frac{R_{L}}{R_{c h}+R_{L}}\right)
\end{aligned}
$$

Another level of approximation on (11) and (18) has also been commonly used and reported in the $\mathrm{THz}$ community as well $[18,26,28]$. Using the academic definition of the drain-tosource current for any FET device, one can find approximately for strong inversion case that: $\partial G_{c h} / \partial V_{g s} \times R_{c h}=1 /\left(V_{g s}-V_{t h}\right)$ and for the weak inversion case that: $\partial G_{c h} / \partial V_{g s} \times R_{c h}=1 / \eta V_{t}$; where $V_{t h}$ is the transistor's threshold voltage, $V_{t}$ is the thermal 
voltage, and $\eta$ is the ideality factor of the subthreshold current. Therefore, an abstract model for $\mathrm{THz}$ rectification at zero-drain biasing condition can be expressed as:

$$
\begin{aligned}
& \Delta V_{d s(\text { load })}^{\text {unbiased }}=\left(\frac{V_{s(a c)}^{2}-V_{d(a c)}^{2}}{4\left(V_{g s}-V_{t h}\right)}\right) \times\left(\frac{R_{L}}{R_{L}+R_{c h}}\right), \quad V_{g s}>V_{t h} \\
& \Delta V_{d s(\text { load })}^{\text {unbiased }}=\left(\frac{V_{s(a c)}^{2}-V_{d(a c)}^{2}}{4 \times \eta V_{t}}\right) \times\left(\frac{R_{L}}{R_{L}+R_{c h}}\right), \quad V_{g s}<V_{t h}
\end{aligned}
$$

Both (19) and (20) can be fitted together using the standard unified charge control model [33]. Comparing the experimental $\mathrm{THz}$ response with this abstract academic model reveals poor matching especially for the strong inversion case as shown in Fig. 7; the experimental results curve in this region is not inversely proportional to $\left(V_{g s}-V_{t h}\right)$ as suggested by (19). As for the weak inversion region, the $\mathrm{THz}$ response is governed only by the loading effect. From that, it is not recommended to use this level of simplifications to describe THz FET response.

As for the drain-biased THz FET responses, Fig. 8 compares the analytical model that was given by (14) with experimental results and they both show great agreement. It is important to notice that the experimental $\mathrm{THz}$ responses were defined as: $\Delta V_{d s(\text { load })}^{\text {biased }}$ (no radiation) - $\Delta V_{d s \text { (load) }}^{\text {biased }}(\mathrm{THz}$ radiation) [29], hence these results are the negative of all the analyses defined in this work. In addition, the normal and exchanged source and drain connections under the same falling $\mathrm{THz}$ radiation enable the examination of localized $\mathrm{THz}$ rectifications at both channel's sides independently. At a very small drain current, symmetric positive and negative results are obtained from this connectivity exchanging which can be explained by replacing $\Delta V_{g s}^{\text {biased }}$ by $\Delta V_{g d}^{\text {bised }}$ in (14) and vice versa. At large drain current values, The THz FET operates in the saturation regime. Therefore, the localized $\mathrm{THz}$ rectifications at one channel's side are solely amplified in the normal connection configuration, while the localized $\mathrm{THz}$ rectifications at the other channel's side are solely amplified in the exchanged connection configuration. Thus, FET edges can subsample the falling $\mathrm{THz}$ radiation with sub-wavelength resolution. Also, multiple adjacent THz FETs can detect and image $\mathrm{THz}$ radiation simultaneously with subwavelength spatial resolution when operating in the saturation regime [43].

\section{CONCLUSION}

The localized $\mathrm{THz}$ rectifications at both source and drain edges of the FET power detectors can be treated logically as miniaturized induced ultrafast Schottky diodes effect. An improved one lumped THz FET power detector model has been presented with additional Schottky diodes at its source and drain sides. This improved one lumped THz FET model is based on the nonlinear analysis of a distributed FET network which is necessary for higher frequencies operation. The developed analytical model works well for a zero-drain biased THz FET detector as well as under any arbitrary biasing conditions. When the THz FET detector operates in the

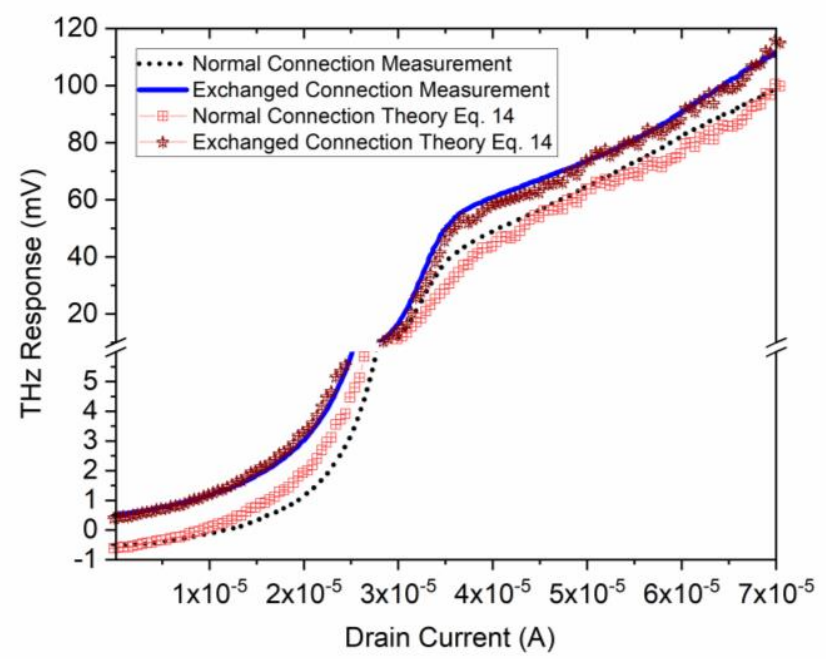

Fig. 8. The measured non-resonant $1.6 \mathrm{THz}$ response of GaAs HEMT with $100 \mathrm{KOhms}$ load resistance as a function of the applied drain current for normal and exchanged source and drain connections under the same falling $\mathrm{THz}$ radiation, as well as the developed nonlinear analytical model given by (14).

saturation regime, the localized $\mathrm{THz}$ rectifications at the source side are being self-amplified by the transistor's gain as in a common source amplifier configuration. A novel analytical expression for the localized $\mathrm{THz}$ rectification at the source side has been developed for THz FET detectors operating in the saturation regime.

\section{REFERENCES}

[1] M. Tonouchi, "Cutting-edge terahertz technology," Nature Photon., vol. 1, no. 2, pp. 97-105, Feb. 2007.

[2] P. H. Siegel, "Terahertz technology", IEEE Trans. Microw. Theory Techn., vol. 50, no. 3. pp. 910-928, Mar. 2002.

[3] I. F. Akyildiz, J. M. Jornet, and C. Han, "TeraNets: Ultra-broadband communication networks in the terahertz band," IEEE Wireless Commun., vol. 21, no. 4, pp. 130-135, Aug. 2014.

[4] J. F. Federici et al., "THz imaging and sensing for security applications-explosives, weapons, and drugs," Semicond. Sci. Technol. vol. 20, no. 7, pp. S266-S280, June 2005.

[5] D. M. Mittleman, "Twenty years of terahertz imaging," Optics Express, vol. 26, no. 8, pp. 9417-9431, Apr. 2018.

[6] K. Ajito and Y. Ueno, "THz chemical imaging for biological applications," IEEE Trans. THz Sci. Technol., vol. 1, no. 1, pp. 293300, Sep. 2011.

[7] Xiang Yang, et al., "Biomedical Applications of Terahertz Spectroscopy and Imaging," Trends Biotechnol. vol. 34, no. 10, pp. 810-824, Oct. 2016.

[8] K. Cooper, et al., "Penetrating 3-D imaging at 4- and 25-m range using a submillimeter-wave radar," IEEE Trans. Microw. Theory Techn., vol. 56, no. 12, pp. 2771-2778, Dec. 2008.

[9] M. Naftaly and R. E. Miles, "Terahertz time-domain spectroscopy for material characterization," Proc. IEEE, vol. 95, no. 8, pp. 1658-1665, Aug. 2007.

[10] D. M. Pozar, Microwave Engineering, 4th ed. Hoboken, NJ: Wiley, 2012.

[11] H. Aghasi et al., "Terahertz electronics: Application of wave propagation and nonlinear processes," Appl. Phys. Rev., vol. 7, no.2, p. 021302, April 2020.

[12] M. Božanić, and S. Sinha, "Emerging Transistor Technologies Capable of Terahertz Amplification: A Way to Re-Engineer Terahertz Radar Sensors," Sensors, vol. 19, no. 11. pp. 2454, May 2019

[13] M. Dyakonov and M. S. Shur, "Shallow water analogy for a ballistic field effect transistor: New mechanism of plasma wave generation by dc current," Phys. Rev. Lett., vol. 71, no. 15, pp. 2465-2468, Oct. 1993. 
[14] M. Dyakonov and M. S. Shur, "Plasma wave electronics: Novel terahertz devices using two dimensional electron fluid," IEEE Trans. Electron Devices, vol. 43, no. 10, pp. 1640-1645, Oct. 1996.

[15] M. Dyakonov and M. Shur, "Detection, mixing, and frequency multiplication of terahertz radiation by two-dimensional electronic fluid," IEEE Trans. Electron Devices, vol. 43, no. 3, pp. 380-387, Mar. 1996.

[16] A. Gutin, T. Ytterdal, V. Kachorovskii, A. Muraviev, and M. Shur, "THz SPICE for modeling detectors and nonquadratic response at large input signal," IEEE Sensors J., vol. 13, no. 1. pp. 55-62, Jan. 2013.

[17] A. Gutin, S. Nahar, M. Hella, and M. Shur, "Modeling terahertz plasmonic Si FETs with SPICE," IEEE Trans. Terahertz Sci. Technol., vol. 3, no. 5, pp. 545-549, Sep. 2013.

[18] A. Lisauskas et al., "Rational design of high-responsivity detectors of terahertz radiation based on distributed self-mixing in silicon fieldeffect transistors," J. Appl. Phys., vol. 105, no. 11, 2009, Art. no. 114511.

[19] Y. Kurita et al., "Ultrahigh sensitive sub-terahertz detection by InPbased asymmetric dual-grating high-electron-mobility transistors and their broadband characteristics," Appl. Phys. Lett., vol. 104, no. 25, pp. 251114-1-251114-4, June 2014.

[20] X. Liu, T. Ytterdal, V. Yu. Kachorovskii, and M. S. Shur, "Compact terahertz SPICE/ADS model,” IEEE Trans. Electron Devices, vol. 66, no.6, pp. 2496-2501, June 2019.

[21] X. Liu et al., "Compact terahertz SPICE model: Effects of drude inductance and leakage," IEEE Trans. Electron Devices, vol. 65, no. 12, pp. 5350-5356, Dec. 2018.

[22] S. Preu et al., "An improved model for non-resonant terahertz detection in field-effect transistors," J. Appl. Phys., vol. 111, no. 2, Jan. 2012, Art. no. 24502.

[23] M. Sakowicz et al., "Terahertz responsivity of field effect transistors versus their static channel conductivity and loading effects," J. Appl. Phys., vol. 110, no. 5, Sep. 2011, Art. no. 54512.

[24] M. Bucher and A. Bazigos, "An efficient channel segmentation approach for a large-signal NQS MOSFET model," Solid-State Electron., vol. 52, no. 2, pp. 275-281, Feb. 2008

[25] Mansun Chan, K.Y. Hui, Chenming Hu, and P.K. Ko "A robust and physical BSIM3 non-quasi-static transient and ac small-signal model for circuit simulation," IEEE Trans. Electron Devices, vol. 45, no. 4, pp. 834-841, Apr. 1998.

[26] E. Ojefors, U. R. Pfeiffer, A. Lisauskas, and H. G. Roskos, "A 0.65 $\mathrm{THz}$ focal-plane array in a quarter-micron CMOS process technology," IEEE J. Solid-State Circuits, vol. 44, no. 7. pp. 19681976, Jul. 2009.

[27] J.-Q. Lu and M. S. Shur, "Terahertz detection by high electron mobility transistor: Enhancement by drain bias," Appl. Phys. Lett., vol. 78, no.17, pp. 2587-2588, Apr. 2001.

[28] D.Veksler, F. Teppe, A. P. Dmitriev, V. Y. Kachorovskii, W. Knap, and M. S. Shur, "Detection of terahertz radiation in gated twodimensional structures governed by dc current," Phys. Rev. B, Condens. Matter, vol.73, no. 12, pp. 125328-1-125328-10, Mar. 2006.

[29] T. A. Elkhatib, V. Y. Kachorovskii, W. J. Stillman, S. Rumyantsev, X.-C. Zhang, and M. S. Shur, "Terahertz response of field-effect transistors in saturation regime," Appl. Phys. Lett., vol. 98, no. 24, Jun. 2011, Art.no. 243505.

[30] M. A. Andersson and J. Stake, "An accurate empirical model based on Volterra series for FET power detectors," IEEE Trans. Microw. Theory Techn., vol. 64, no. 5, pp. 1431-1441, May 2016.

[31] M. I. W. Khan et al., "Nonlinear analysis of nonresonant THz response of MOSFET and implementation of a high-responsivity cross-coupled THz detector," IEEE Trans. THz Sci. Technol., vol. 8, no. 1, pp. 108120, Jan. 2018.

[32] P. F"oldesy, "Terahertz responsivity of field-effect transistors under arbitrary biasing conditions," J. Appl. Phys., vol. 114, no. 11, Sep. 2013, Art.no. 114501

[33] T. A. Elkhatib et al., "Enhanced plasma wave detection of terahertz radiation using multiple high electron-mobility transistors connected in series," IEEE Trans. Microw. Theory Techn., vol. 58, no. 2, pp. 331339, Feb. 2010.

[34] J. D. Sun et al., "High-responsivity, low-noise, room-temperature, self-mixing terahertz detector realized using floating antennas on a GaN-based field-effect transistor," Appl. Phys. Lett., vol. 100, no. 1, p. 013506, Jan. 2012.
[35] J. D. Sun et al., "Probing and modelling the localized self-mixing in a GaN/AlGaN field-effect terahertz detector," Appl. Phys. Lett., vol. 100, no. 17, p. 173513, Apr. 2012.

[36] Q. Yang et al., "Improved performance of CMOS terahertz detectors by reducing MOSFET parasitic capacitance," IEEE Access, vol. 7, pp. 9783-9789, Dec. 2019.

[37] M. Woo Ryu et al., "High-performance of plasmonic THz detector based on asymmetric FET with vertically integrated antenna in CMOS technology," IEEE Trans. Electron. Devices, vol. 63, no. 4, pp. 17421748, Feb. 2016

[38] W. Knap et al., "Field effect transistors for terahertz detection: Physics and first imaging applications," J. Infr., Millim., Terahertz Waves, vol. 30, no. 12, pp. 1319-1337, Aug. 2009.

[39] S. Rosenblatt, et al., "Mixing at $50 \mathrm{GHz}$ using a single-walled carbon nanotube transistor," Appl. Phys. Lett., vol. 87, no. 15, p. 153111, Oct. 2005.

[40] R. Tan, et al., "Modeling an antenna-coupled graphene field-effect terahertz detector," Appl. Phys. Lett., vol. 103, no.17, pp. 173507, Oct. 2013.

[41] L. Vicarelli et al., "Graphene field-effect transistors as roomtemperature terahertz detectors," Nature. Materials., vol. 11, pp. 865871, Sep. 2012.

[42] S. Boppel et al., "CMOS integrated antenna-coupled field-effect transistors for the detection of radiation from 0.2 to $4.3 \mathrm{THz}$," IEEE Trans. Microw. Theory Techn., vol. 60, no. 12, pp. 3834-3843, Dec. 2012.

[43] T. A. Elkhatib, A. V. Muravjov, D. B. Veksler, W. J. Stillman, X.-C. Zhang, M. S. Shur, and V. Y. Kachorovskii, "Subwavelength detection of terahertz radiation using GaAs HEMTs," in Sensors, 2009 IEEE, 2009, pp. 1988-1990.

[44] W. Stillman, M. S. Shur, D. Veksler, S. Rumyantsev, and F. Guarin, "Device loading effects on nonresonant detection of terahertz radiation by silicon MOSFETs," Electron. Lett., vol. 43, no. 7, pp. 422-423, Mar. 2007.

[45] U. Pfeiffer, C. Mishra, R. Rassel, S. Pinkett, and S. Reynolds, "Schottky barrier diode circuits in silicon for future mmWave and $\mathrm{THz}$ applications," IEEE Trans. Microw. Theory and Tech., vol. 56, no. 2, pp. 364-371, Feb. 2008.

[46] D. B. Veksler, V. Y. Kachorovskii, A. V. Muravjov, T. A. Elkhatib, K. N. Salama, X.-C. Zhang, and M. S. Shur, "Imaging of field-effect transistors by focused terahertz radiation," Solid-State Electron., vol.53, no. 6, pp. 571-573, Jun. 2009.

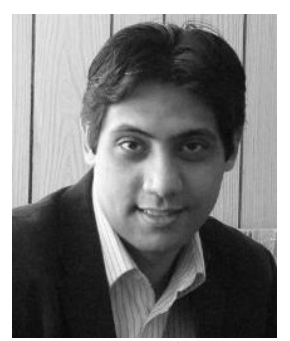

Tamer A. Elkhatib received the B.Sc. degree in electronics and communication and the M.Sc. degree in engineering mathematics and physics (both with honors) from Cairo University, Cairo, Egypt, in 2002 and 2006, respectively, and the Ph.D. degree in electrical engineering from Rensselaer Polytechnic Institute, Troy, NY, USA, in 2010. He was a Senior Scientist with Microsoft, Mountain View, CA, USA, developing the next-generation time-of-flight image sensor technology for XboxOne (Kinect 2) and HoloLens from 2010 till 2017. $\mathrm{He}$ is currently an Assistant Professor at Faculty of Engineering, Cairo University, Egypt. He is the author and coauthor of 20 scientific articles published in refereed journals and conference proceedings. He received the "Walter Kosonocky" Best Paper Award from the international image sensor society in 2017. Dr Elkhatib holds 6 issued US patents. His current research interests include semiconductor device modeling and simulation, Terahertz electronics and imaging, CMOS image sensors, Time-of-flight sensors. 